\title{
Foreword to the Special Issue on Applied Earth Observation and Remote Sensing in India
}

I NDIA is at the forefront in several areas of research related to Earth observation and remote sensing. Its space program has been in the news often in recent times, but other aspects of Indian research in geosciences and remote sensing remain relatively unknown to the international community of researchers in the field. It is the objective of this special issue of IEEE JOURNAL OF SELECTED TOPICS IN APPLIED EARTH OBSERVATIONS AND REMOTE SENSING (IEEE JSTARS)_- "Applied Earth Observation and Remote Sensing in India," to highlight the work of Indian scientists in these areas. A call for papers was announced during November 2016. In response, 60 manuscripts were received from variety of fields. Out of these, 17 papers were selected for publication.

\section{EARTH ObSERVATION AND REMOTE SENSING IN INDIA}

The importance of Earth observation and remote sensing in India cannot be overstated. From crop management to defense, astronomy to weather prediction, satellites provide vital information to Indian scientists, and operational agencies for the benefit of society. India's initiatives in Earth observation, remote sensing, and recently planetary and astrophysical missions have spurred broad innovations in design and development of sensors, pre/post processing and analyzes of data acquired, and a host of applications in communications, defense, agricultural monitoring, oceanographic studies, and disaster response, to name a few.

The first satellite designed and fabricated by India, Aryabhatta, was launched on April 19, 1975. Since then, around 50 satellites have been successfully launched. The instruments on these satellites range from X-ray sensors to hyperspectral imagers, and are placed both in Sun-synchronous and Geostationary orbits. Details of all the satellites launched can be found in [items 1), 2), and 3) in the Appendix]. Of special mention are the data acquired by the Cartosat and Resourcesat series: The data from these satellites have revolutionized the field of digital mapping in India. In the last decade, as a first step toward outer space exploration, India launched Chandrayaan-1 [item 4) in the Appendix], subsequently the Mangalayaan Mars Orbiter Mission [item 5) in the Appendix], and Astrosat, a mission to map Earth-like planets. The Indian Space Research Organisation (ISRO) has partnered with the National Aeronautics and Space Administration (NASA) on a number of missions, including Chandrayaan-1, scatterometry missions for measuring ocean winds, and most recently in developing an Earth observing science and applications mission that will exploit synthetic aperture radar (SAR). This mission, known as the NISAR Mission, is proposed to launch in late 2021 [item 6) in the Appendix]. ISRO has also partnered with other space agencies such as the French Space Agency.

Digital Object Identifier 10.1109/JSTARS.2017.2773847
Beyond the scientific successes, data from these satellites have been used at various stages of operational monitoring and policy making by the Indian government. In addition, the use and analysis of these data support the research by academicians across India. This issue includes some of these works at the forefront of their research field.

\section{IEEE GEOSCIENCES AND REMOTE SENSING SOCIETY (GRSS) IN INDIA}

The geophysical diversity of the Indian subcontinent and India's large population make it both necessary and challenging to develop techniques that respond to scientific and societal needs, with a number of problems unique to the subcontinent. Historically, academicians from India have not been involved to a wide extent in the International remote sensing community. This is poised to change with the new initiatives of the Advisory Committee (AdCom) of IEEE Geosciences and Remote Sensing Society (GRSS) to foster the participation of India's scientists and engineers working in remote sensing in IEEE GRSS activities worldwide. Several local GRSS chapters have been established in India [item 7) in the Appendix] include GRSS Delhi [item 8) in the Appendix], GRSS Kolkata [items 9) and 10) in the Appendix], GRSS Bangalore [item 11) in the Appendix], GRSS Gujarat [item 12) in the Appendix], GRSS Bombay, and GRSS Hyderabad Chapters. With the establishment of these Indian GRSS chapters, many young authors whose papers have been accepted for IGARSS have been provided with travel grant awards. IEEE GRSS has also supported organizing several short courses and seminars by distinguished lecturers across the chapters in India. It is the express intent of GRSS to create a strong presence of GRSS in India to increase the visibility of the work being done in India, and to foster stronger international collaborative efforts.

\section{ORgAniZATION OF THE SPECIAL ISSUE}

The 17 articles selected for publication are organized into the following three groups:

1) Microwave sensing-based studies (3)

2) Algorithms and frameworks (8)

3) Remote sensing applications (6)

In Group 1, there are three papers that use polarimetric SAR data. The first two papers use a nonstandard basis to classify surfaces. In the first paper, datasets from RISAT-1 acquired over Mumbai city are analyzed by using a decomposition technique that is based on an unsupervised model (A. Kumar et al.). In the second paper, scattering powers for the two structurally distinct crops are analyzed (V. Kumar et al.). The third paper uses C-Band Radarsat-2 spaceborne SAR data in a polarimetric SAR tomographic inversion (PolTomSAR) to estimate the vertical 
distribution of scattering centers in a forested area (S. Kumar et al.).

In Group 2, the papers rely primarily on optical data, and apply sophisticated statistical and machine-learning methods to classification and interpretation. In the first paper, using Bayesian techniques, Level-2 Aerosol Optical Depth (AOD) corresponding to three locations that have long-term data (for the period between 2001-2014), retrieved from Multi-angle Imaging SpectroRadiometer (MISR) and Moderate Resolution Imaging Spectroradiometer (MODIS) satellite data, is merged in conjunction with AErosol RObotic NETwork (AERONET) data (Singh et al.). In the second paper of this group, a progress granular neural network-based classification approach developed by the authors is employed for classification of land use/land cover features from both multispectral and hyperspectral remote sensing images (D. Kumar et al.). In the third paper, an open issue of hyperspectral image classification with sparse labeled samples is addressed through a technique exploiting dimensionality reduction, spectral-spatial information and classification with active learning (Patra et al.). The fourth paper exploits a deep-learningbased framework, involving an ensemble of multiple forecasting modules to address the problem of missing data in remote sensing time series analysis. This framework would reconstruct the missing data further facilitating the analysis of remote sensing time series (Das and Ghosh). In the fifth paper, hyperspectral images are segmented by following a $k$-means clustering approach, and by following mutual interest neighbor information, segmented regions are merged in a hierarchical manner (Mehta and Dikshit). In the sixth paper, a methodology based on fractal dimension techniques is applied to identify normalized difference vegetation index (NDVI) profile patterns further to reveal patterns that would assist discrimination of different classes. The two ad hoc methods, namely box counting and rescaled range methods, are employed to show applications in the context of mapping vegetation phenomena from remotely sensed data. The seventh paper focuses on extracting information from time series NDVI data (at coarse spatial resolution) and testing fractal methods for pattern characterization (Chockalingam and Mondal). In the eighth paper, to denoise seismic attributes further to improve the porosity column, a diffusion filter-based scheme is proposed and demonstrated (Chaki et al.).

In Group 3, there are six papers dealing with science and applications. The first paper maps superglacial lakes (SGLS) of Gangotri glacier using high resolution LISS-IV data. The work uses an object-based image analysis approach, and an index is proposed for classifications of SGLs (Mitkari et al.). In the second paper in this group, Prakash and Nagarajan provide a detailed inventory of the spatial distribution and temporal changes of glacial lakes situated in and around the northwestern Himalaya region, recorded from Corona, ASTER, and IRS-R2 data of the years 1971, 2003, and 2011. In the third paper, a geodetic mass budget and ice velocity patterns of Gangotri glacier, one of the largest Himalayan glaciers, are estimated using optical satellite data, further analyzing the spatial distribution of ice thickness and possible locations of glacial lake formation. It is discovered that mass loss is increasing with reducing velocity patterns of the Gangotri glacier (Bhushan et al.). In the fourth paper, an algorithm to detect the fog over India from INSAT-3D imagery in a real-time mode is demonstrated (Chaurasia and Jenamani). In the fifth paper, aerosol optical depth over northwest Himalayan region is examined and assessed through ground-based measurements and MODIS and MISR satellite data (Shaik et al.). And finally, an approach to agriculture monitoring at regional scales is proposed by integrating drone data with remotely sensed data. The novelty of this work lies in developing a methodology that may be able to distinguish between sparse and dense vegetation within a field by using satellite and drone data (Murugan et al.).

A special issue, with its demanding solicitation, review, and production schedule, can only sample the work of the community. These seventeen papers, though only a sample, demonstrate the breadth of research being conducted in India, and the promise of a community addressing important scientific issues using cutting edge methods.

\section{ACKNOWLEDGMENT}

Following the hard deadlines and tight time-schedules from the phase of submitting the special issue proposal to the release of the special issue is indeed a herculean task. This task was achieved mainly due to the help of the Reviewers, EditorIn-Chief of IEEE JSTARS, Prof. Q. Du, and the authors who have patiently addressed all the queries of the Reviewers/Guest Editors/E-i-C. We gratefully acknowledge all their support. This India special issue is the brainchild of Prof. W. Emery, IEEE Publication Committee Chair. The guest editors are also grateful to the members of the IEEE GRSS AdCom.

\section{AVIK BHATTACHARYA, Guest Editor \\ Centre of Studies in Resources Engineering Indian Institute of Technology Bombay Mumbai 400076, India}

LORENZO BRUZZONE, Guest Editor

Department of Information Engineering and Computer Science

University of Trento

Trento 38123, Italy

\section{B. S. DAYA SAGAR, Guest Editor \\ Systems Science and Informatics Unit \\ Indian Statistical Institute \\ Bangalore 560059, India}

PAUL A. RoSEN, Guest Editor
Jet Propulsion Laboratory
California Institute of Technology
Pasadena, CA 91109 USA

\section{APPENDIX \\ RELATED WORK}

1) [Online]. Available: https://www.isro.gov.in/missions-0. Accessed on: Nov. 1, 2017.

2) P. Murugan, "Indian remote sensing missions and payloads-A glance,' IRS-Programme Management Office, ISRO Satellite Centre, Bangalore, India, p. 298, 2016.

3) A. S. Kirankumar and T. Misra, “A glimpse of ISRO's EO programme," IEEE Geosci. Remote Sens. Mag., vol. 3, no. 4, pp. 46-53, Dec. 2014.

4) M. Annadurai and T. K. Alex, "Chandrayaan-1: An update," J. Aerosp. Sci. Technol., vol. 61, no. 1, pp. 81-87, 2009.

5) M. Kramer, "Liftoff! India's first mars probe launches toward the red planet," Nov. 2013. [Online]. Available: 
http://www.space.com/23464-india-launches-marsorbiter-mission.html. Accessed on: Oct. 31, 2017.

6) P. A. Rosen, Y. Kim, R. Kumar, T. Misra, R. Bhan, and V. R. Sagi, "Global persistent SAR sampling with the NASA-ISRO SAR (NISAR) mission,” in Proc. IEEE Radar Conf., 2017, pp. 410-414.

7) “GRSS chapters and contact information," IEEE Geosci. Remote Sens. Mag., vol. 5, no. 3, pp. 63-64, Sep. 2017.

8) "GRSS activities by the IEEE GRSS Delhi chapter," IEEEE Geosci. Remote Sens. Mag., vol. 3, no. 2, pp. 55-57, Jun. 2015.
9) A. Maitra, "IEEE geoscience and remote sensing society: Kolkata chapter report," IEEEE Geoscience Remote Sens. Mag., vol. 3, no. 3, pp. 123-125, Sep. 2015.

10) A. Maitra, "A profile of remote-sensing activities in the Kolkata chapter 2015-2016," IEEEE Geosci. Remote Sens. Mag., vol. 5, no. 2, pp. 97-100, Jun. 2017.

11) B. S. D. Sagar and S. K. Meher, "Bangalore section chapter of the GRSS," IEEE Geosci. Remote Sens. Mag., vol. 1, no. 2, pp. 72-73, Jun. 2013.

12) "IEEE Gujarat section geoscience and remote sensing society-Chapter," Newsletter, vol. 1, no. 1, 2013.

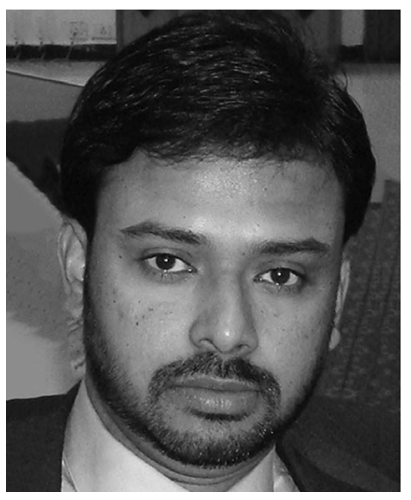

Avik Bhattacharya (M'08-SM'16) received the M.Sc. degree in mathematics from the Indian Institute of Technology Kharagpur, Kharagpur, India, in 2000, and the Ph.D. degree in remote sensing image processing and analysis from Télécom ParisTech, Paris, France, and the Ariana Research Group, Institut National de Recherche en Informatique et en Automatique, Sophia Antipolis, Nice, France, in 2007.

He is currently an Associate Professor at the Centre of Studies in Resources Engineering, Indian Institute of Technology Bombay (CSRE, IITB), Mumbai, India. Before joining IITB, he was a Canadian Government Research Fellow at the Canadian Centre for Remote Sensing (CCRS) in Ottawa, ON, Canada. He received the Natural Sciences and Engineering Research Council of Canada visiting scientist fellowship at the Canadian national laboratories, from 2008 to 2011. His current research interests include SAR polarimetry, statistical analysis of polarimetric SAR images, machine learning, and pattern recognition.

Dr. Bhattacharya is an Associate Editor of the IEEE GEOSCIENCE AND REMOTE SENSING LETTERS. He is the Founding Chairman of the IEEE Geoscience and Remote Sensing Society of the Bombay chapter. He is currently leading the Microwave Remote Sensing Lab (www.mrslab.in) at CSRE, IITB.

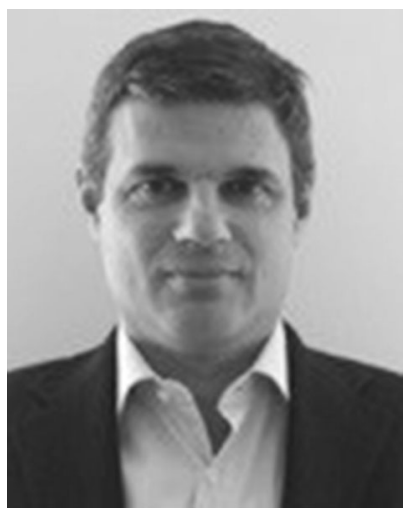

Lorenzo Bruzzone (S'95-M'98-SM'03-F'10) received the Laurea (M.S.) degree in electronic engineering (summa cum laude) and the Ph.D. degree in telecommunications from the University of Genoa, Italy, in 1993 and 1998, respectively.

He is currently a Full Professor of telecommunications at the University of Trento, Trento, Italy, where he teaches remote sensing, radar, and digital communications. He is the Founder and the Director of the Remote Sensing Laboratory, Department of Information Engineering and Computer Science, University of Trento. Among the others, he is the Principal Investigator of the Radar for icy Moon exploration instrument in the framework of the JUpiter ICy moons Explorer mission of the European Space Agency. He is the author (or co-author) of 203 scientific publications in referred international journals (148 in IEEE journals), more than 280 papers in conference proceedings, and 21 book chapters. He is editor/co-editor of 18 books/conference proceedings and one scientific book. His papers are highly cited, as proven from the total number of citations (more than 20 000) and the value of the h-index (69) (source: Google Scholar). He was invited as keynote speaker in more than 30 international conferences and workshops. His current research interests include the areas of remote sensing, radar and SAR, signal processing, and pattern recognition. He promotes and supervises research on these topics within the frameworks of many national and international projects. He is the Principal Investigator of many research projects.

Dr. Bruzzone has been a member of the Administrative Committee of the IEEE Geoscience and Remote Sensing Society (GRSS), since 2009. He ranked first place in the Student Prize Paper Competition of the 1998 IEEE International Geoscience and Remote Sensing Symposium (IGARSS), Seattle, WA, USA, in July 1998. Since that, he was recipient of many international and national honors and awards, including the recent IEEE GRSS 2015 Outstanding Service Award and the 2017 IEEE IGARSS Symposium Prize Paper Award. He was a Guest Co-Editor of many Special Issues of international journals. He is the Co-Founder of the IEEE International Workshop on the Analysis of Multi-Temporal Remote-Sensing Images series and is currently a member of the Permanent Steering Committee of this series of workshops. Since 2003, he has been the Chair of the SPIE Conference on Image and Signal Processing for Remote Sensing. Since 2013, he has been the founder Editor-in-Chief of the IEEE GEOSCIENCE And Remote SENSING MAGazine. He is currently an Associate Editor for the IEEE TRANSACTIONS ON GEOSCIENCE AND REMOTE SENSING. He has been a Distinguished Speaker of the IEEE Geoscience and Remote Sensing Society between $2012-2016$. 


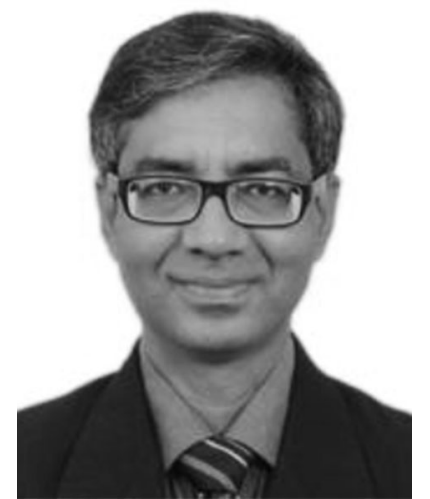

B. S. Daya Sagar (M'03-SM'03) received the M.Sc. and Ph.D. degrees in geoengineering and remote sensing from the Faculty of Engineering, Andhra University, Visakhapatnam, India, in 1991 and 1994, respectively.

He is a Full Professor of the Systems Science and Informatics Unit (SSIU) at the Indian Statistical Institute, Bangalore, India. He is also the first Head of the SSIU. Earlier, he worked in College of Engineering, Andhra University, and Centre for Remote Imaging Sensing and Processing (CRISP), The National University of Singapore in various positions during 19922001. He was an Associate Professor and a Researcher in the Faculty of Engineering \& Technology (FET), Multimedia University, Malaysia during 2001-2007. Since 2017, he has been a Visiting Professor at the University of Trento, Trento, Italy. His research interests include mathematical morphology, GISci, digital image processing, fractals and multifractals their applications in extraction, analyzes, and modeling of geophysical patterns. He has published more than 80 papers in journals, and has authored and/or guest edited 11 books and/or special theme issues for journals. He recently authored a book entitled Mathematical Morphology in Geomorphology and GISci (CRC Press, 2013, p. 546).

Prof. Sagar recently co-edited a special issue on "Filtering and Segmentation with Mathematical Morphology" for IEEE JOURNAL ON SElected Topics IN Signal PRocessing (vol. 6, no. 7, pp. 737-886, 2012). He is an elected Fellow of Royal Geographical Society (1999), Indian Geophysical Union (2011), and was a member of New York Academy of Science during $1995-1996$. He received Dr. Balakrishna Memorial Award from Andhra Pradesh Akademi of Sciences in 1995, Krishnan Gold Medal from Indian Geophysical Union in 2002, and "Georges Matheron Award-2011 (with Lecturership)" of International Association for Mathematical Geosciences. He is the Founding Chairman of Bangalore Section IEEE GRSS Chapter. He is on the Editorial Boards of Computers \& Geosciences, and Frontiers: Environmental Informatics.

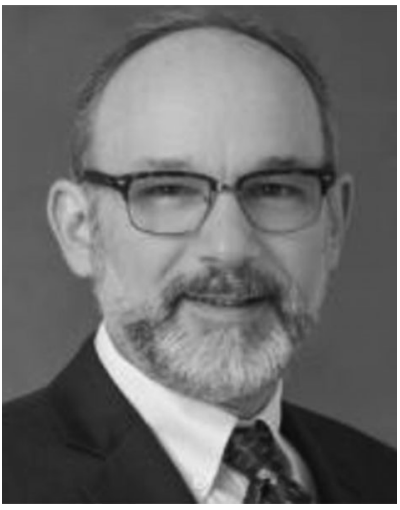

Paul A. Rosen (F'11) received the B.S. and M.S. degrees from the University of Pennsylvania, Philadelphia, PA, USA, in 1981 and 1982, respectively, and the Ph.D. degree from the University of Stanford, Stanford, CA, USA, in 1989, all in electrical engineering.

He is currently a Project Scientist for NASA-ISRO synthetic aperture radar mission concept at the Jet Propulsion Laboratory. He was the Shuttle Radar Topography Mission (SRTM) Project Element Manager for Algorithm Development and Verification from 1996 to 2000 and was the SRTM metrology Tiger Team Lead in 2001. Until 2012, he was the manager of the Radar Science and Engineering Section and Project Scientist for the DESDynI Mission Concept at the Jet Propulsion Laboratory. His assignments at JPL have centered on scientific and engineering research and development of radar instruments and applications. Prior to JPL, he spent two years at Kanazawa University, Kanazawa, Japan. He is a visiting faculty member and a Lecturer at the Division of Geological and Planetary Sciences at Caltech, and has served on the UCLA Extension Program faculty. He is an author of more than 45 journal and book chapter publications, and more

than 100 conference papers.

Dr. Rosen was a team leader on the Shuttle Radar Topography Mission, for which he received NASA's Exceptional Service Medal (2001) and NASA's Exceptional Achievement Medal (2002). He is a member of the American Geophysical Union. 